\title{
Making sense of reAKTive oxygen species
}

\author{
GM DeNicola ${ }^{*, 1}$ and IS Harris ${ }^{*, 2}$ \\ Cell Death and Differentiation (2016) 23, 1269-1270; doi:10.1038/cdd.2016.57; published online 17 June 2016
}

The association of cellular redox states with a variety of physiological and pathophysiological states is becoming increasingly appreciated in biology. Recent studies report on advances in our ability to measure redox changes and exploit them for therapeutic benefit.

The investigation of changes in cellular redox is technically challenging. Redox couples, such as GSH:GSSG or NADPH: NADP+, change dynamically in response to oxidative challenges and the ratio of these molecules can vary significantly in subcellular compartments. The development of genetically encoded biosensors, which give a read-out of hydrogen peroxide or the GSH:GSSG redox couple in real time, has advanced our ability to assess changes in redox homeostasis within the cell. However, existing redox biosensors have their limitations-while they are able monitor changes in the antioxidant state after oxidative insults, they lack the sensitivity required to measure basal, unstimulated $\mathrm{H}_{2} \mathrm{O}_{2}$ levels. To overcome these challenges, Morgan et al. ${ }^{1}$ developed a new ultrasensitive, genetically encoded $\mathrm{H}_{2} \mathrm{O}_{2}$ sensor based on a redox relay between Tsa2, a 2-Cys peroxidredoxin from Saccharomyces cerevisiae, and roGFP2, a redox-sensitive fluorescent probe. The authors observed that existing sensors, such as $\mathrm{HyPer}^{2}$ and roGFP2-Orp1, ${ }^{3}$ react with $\mathrm{H}_{2} \mathrm{O}_{2}$ 100-fold more slowly than 2-Cys peroxiredoxins, the most efficient thiol peroxidases. By screening all eight yeast thiol peroxidases for their ability to transfer electrons from $\mathrm{H}_{2} \mathrm{O}_{2}$ to roGFP2, the authors found that the roGFP2-Tsa2 fusion showed greater and more prolonged roGFP2 oxidation after $\mathrm{H}_{2} \mathrm{O}_{2}$ exposure than the existing roGFP2-Orp1 sensor.

The authors found that the roGFP2-Tsa2 sensor demonstrated the greatest amplitude change in response $\mathrm{H}_{2} \mathrm{O}_{2}$, but its sensitivity was limited by thioredoxin-mediated reduction of Tsa2, as Trx1 and Trx2 compete with roGFP2 for oxidizing equivalents from the Tsa2 moiety. To resolve this issue, a mutation was engineered $\left(\Delta C_{\mathrm{R}}\right.$-in which the resolving cysteine of Tsa2 was mutated) to prevent Trx-mediated reduction of roGFP2-Tsa2, resulting in not only a $20 \times$ increased sensitivity than HyPer and roGFP2-Orp1, but also in the ability to monitor the basal oxidation state of the cell. In addition, hyperoxidation and loss of oxidation response can limit the utility of sensors at high $\mathrm{H}_{2} \mathrm{O}_{2}$ levels. The authors found that the roGFP2-Tsa2 fusion was insensitive to hyperoxidation up to $1 \mathrm{mM} \mathrm{H} \mathrm{O}_{2}$, suggesting it is unlikely to be inactivated due to hyperoxidation in the physiological $\mathrm{H}_{2} \mathrm{O}_{2}$ range. In addition, the ideal sensor should have as little effect on the redox state of the cells as possible. The authors mutated the peroxidatic cysteine on Tsa2 $\left(\Delta C_{\mathrm{p}}\right)$ and found that roGFP2-Tsa2 $\Delta C_{\mathrm{p}} \Delta C_{\mathrm{R}}$ was catalytically inactive and did not affect cellular peroxidase activity or endogenous $\mathrm{H}_{2} \mathrm{O}_{2}$ homeostasis.

Next, Morgan et al. targeted the sensor to both the mitochondria and cytosol to investigate compartmentspecific oxidation states of the cell. By using yeast respiratory chain mutants, the authors found that mitochondrial matrix $\mathrm{H}_{2} \mathrm{O}_{2}$ levels increased without a corresponding rise in cytosolic $\mathrm{H}_{2} \mathrm{O}_{2}$ levels. However, $\mathrm{H}_{2} \mathrm{O}_{2}$ treatment caused an increase in both cytosolic and matrix $\mathrm{H}_{2} \mathrm{O}_{2}$ oxidation states. The authors conclude that although $\mathrm{H}_{2} \mathrm{O}_{2}$ diffuses freely from the cytosol and mitochondrial matrix, mitochondrial matrix $\mathrm{H}_{2} \mathrm{O}_{2}$ levels can be altered without affecting overall cytosolic $\mathrm{H}_{2} \mathrm{O}_{2}$ levels. Importantly, high matrix $\mathrm{H}_{2} \mathrm{O}_{2}$ levels were shown to limit cellular growth rate.

Ultrasensitive $\mathrm{H}_{2} \mathrm{O}_{2}$ sensors that can be targeted to subcellular compartments have utility in many biological contexts. In an independent study by Dey et al., ${ }^{4}$ the roGFPOrp1 and Grx1-roGFP sensors were used in cardiomyocytes to examine the relative contributions of the thioredoxin- and glutathione-dependent antioxidant systems to $\mathrm{H}_{2} \mathrm{O}_{2}$ dynamics in both the cytosol and mitochondria. Instead of only investigating changes in oxidation states after oxidative insults, the authors interrogated these oxidative stresses after depletion of important antioxidant systems within the mitochondria and cytosol. They found that perturbing the NADPH-generating enzymes in the mitochondria increased ROS in the cytosol, but not vice versa. These findings argue that the mitochondria function as an ROS sink due to both high NADPH generation and $\mathrm{GSH}$ reduction. In addition, impairment of mitochondrial ROS scavenging increased cytosolic ROS during an exogenous $\mathrm{H}_{2} \mathrm{O}_{2}$ challenge. However, it was unclear whether mitochondria were reducing ROS directly, or whether mitochondrial NADPH was shuttled to the cytosol to support cytosolic antioxidant defense. Interestingly, these findings are in contrast to what was observed by Morgan et al. in yeast,

\footnotetext{
${ }^{1}$ Department of Cancer Imaging and Metabolism, Moffitt Cancer Center and Research Institute, Tampa, FL 33612, USA and ${ }^{2}$ Ludwig Center, Harvard Medical School, Boston, MA 33612, USA

*Corresponding author: Dr IS Harris, Ludwig Center, Harvard Medical School, Boston, MA 02115, USA. Tel: 857234 8624; Fax: 617432 3969;

E-mail: Isaac_harris@hms.harvard.edu

or GM DeNicola, Department of Cancer Imaging and Metabolism, Moffitt Cancer Center and Research Institute, Tampa, FL 33612, USA.Tel: 8137458371 ; Fax: 813745 3829; E-mail: Gina.DeNicola@moffitt.org
} 


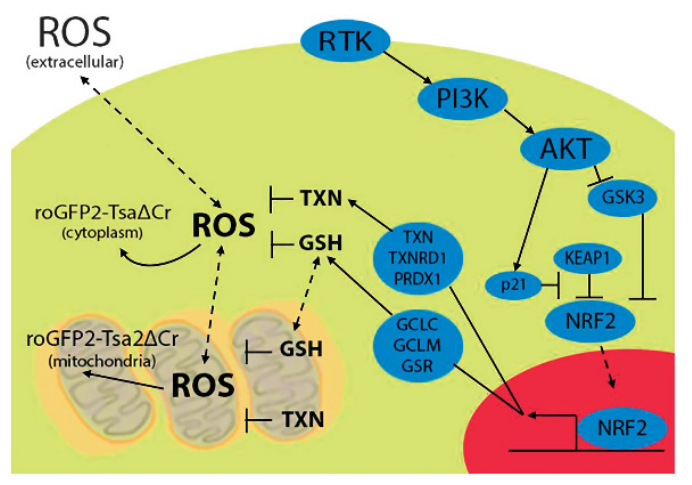

Figure $1 \mathrm{PI} 3 \mathrm{~K} / \mathrm{AKT}$ signaling modulates the cellular redox state. Activation of AKT via receptor tyrosine kinases (RTK) or PI-3-kinase (PI3K) leads to NRF2 stabilization via inhibition of GSK3 $\beta$ and accumulation of p21, which inhibits KEAP1 binding to NRF2. NRF2 accumulates and translocates to the nucleus where it transactivates antioxidant response genes including glutathione biosynthesis enzymes (GCLC, GCLM and GSR) and enzymes that reduce and utilize the protein antioxidant thioredoxin (TXN, TXNRD1 and PRDX1) to control cellular ROS. These antioxidant systems function in both the cytosol and mitochondria. Sensors such as roGFP2-Tsa $\Delta \mathrm{Cr}$ targeted to the cytoplasm and mitochondrial compartments can be used to interrogate the compartmentalization of ROS following PI3K/AKT activation or other signaling events, providing greater insight into how signaling modulates the cellular redox state

although the methods of perturbing metabolism were very different, and may vary by cell type, context or organism.

The interplay of metabolic and antioxidant systems is only beginning to be uncovered. ${ }^{5}$ The studies from Morgan et al. and Dey et al. nicely demonstrate that endogenous changes in subcellular redox couples and ROS can be monitored in response to perturbations in growth and cellular metabolism. The ability to monitor the effects of endogenous redox changes will help answer an outstanding question in the field of cancer research-why are cells with oncogenic pertubations so dependent on glutathione and other antioxidant systems?

In a recent report in Nature Cell Biology, Lien et al. ${ }^{6}$ demonstrate that activating mutations in the PI3K/Akt pathway drive an increase in GSH synthesis, rendering them susceptible to inhibition of this antioxidant. Metabolic profiling of breast cancer cells expressing either a wild-type or a constitutively active Akt2 was performed and they found that glutathione was one of the most differentially expressed metabolites in the Akt2 mutant cancer cells. Importantly, Akt2- mutated cells were resistant to a variety of oxidative insults due to an increased buffering capacity. In addition, blocking GSH synthesis with the chemical compound buthionine sulfoximine (BSO) prevented growth in 3D culture and colony formation of Akt-mutated breast epithelial cells. This result parallels previous reports showing that GSH is essential for initiation of breast cancer malignancies. ${ }^{7}$ The authors sought to exploit this dependence on glutathione for therapeutic gain and found that reducing GSH synthesis sensitized breast cancer cells to cisplatin both in cell culture and in established tumors.

Lien and colleagues then examined the mechanism by which mutant Akt2 regulates glutathione synthesis and found it was dependent on p21/GSK3-mediated stabilization of the antioxidant transcription factor NRF2. Furthermore, they identified differential regulation of NRF2 target genes not only in normal mammary epithelial cells with hyperactivation of the PI3K/Akt pathway, but also in human breast tumors.

Taken together, these reports highlight the need for a multidisciplinary approach to studying antioxidant systems (Figure 1). The development of redox-sensitive versions of other fluorescent proteins, such as RFP, will allow for the simultaneous measurement of multiple redox couples within different cellular compartments. Additionally, the combination of redox sensors with metabolic sensors, such as the NADH sensor Peredox, ${ }^{8}$ will shed light on the interplay between redox couples and cellular systems. A more in-depth characterization of the effect of oncogene hyperactivation on compartment-specific redox states will advance our understanding of cancer progression and provide avenues for therapeutic intervention.

\section{Conflict of Interest}

The authors declare no conflict of interest.

Acknowledgements. We thank Jonathan Coloff and Maria Angelica Martinez Gakidis for scientific editing of the manuscript and Tamara Lutchman for creation of the illustration in the manuscript.

\footnotetext{
1. Morgan B et al. Nat Chem Biol 2016; doi:10.1038/nchembio.2067.

2. Belousov VV et al. Nat Methods 2006; 3: 281-286.

3. Gutscher M et al. Nat Methods 2008; 5: 553-559.

4. Dey S et al. J Biol Chem 2016; 291: 11185-11197.

5. DeNicola GM et al. Nat Genet 2015; 47: 1475-1481.

6. Lien EC et al. Nat Cell Biol 2016; doi:10.1038/ncb3341.

7. Harris IS et al. Cancer Cell 2015; 27: 211-222.

8. Hung YP et al. Cell Metab 2011; 14: 545-554.
} 